\title{
FOOTBRIDGE DYNAMIC PERFORMANCE ASSESSMENT USING INERTIAL MEASUREMENT UNITS
}

\author{
James M.W. BROWNJOHN \\ Professor \\ University of Exeter \\ Exeter, United Kingdom \\ j.brownjohn@exeter.ac.uk
}

\author{
Mateusz BOCIAN \\ Lecturer \\ University of Leicester \\ Leicester, United Kingdom \\ m.bocian@leicester.ac.uk
}

\author{
David HESTER \\ Lecturer \\ Queen's University Belfast \\ Belfast, United Kingdom \\ d.hester@qub.ac.uk
}

\section{Summary}

Dynamic performance of footbridges is still a great concern to designers, operators and users, with many structures requiring investigation before, during and after construction to manage performance.

We have been investigating the use of wireless inertial measurement units (IMUs) designed for biomechanics, health and sports science application for estimating human dynamic loads or ground reaction forces (GRFs) on structures. The aim has been to move from direct measurements using force plates and treadmills, via optical motion capture in the laboratory (with application of Newton's Second law), to unconstrained field conditions. Initially we used IMUs to evaluate pedestrian synchronisation, but we found that a single IMU attached to the $\mathrm{C} 7$ neck vertebra can provide a remarkably accurate estimate of vertical GRF. With an ability to communicate and synchronise within a group wirelessly, to identify orientation and transform accelerations into world coordinates, IMUs can identify both the GRF force vectors and their time varying location with a moving pedestrian. As a side-benefit, the signal to noise ratio and synchronisation accuracy are sufficient to enable low-cost wireless footbridge ambient vibration testing and monitoring. So far we have used IMUs for ambient and forced vibration testing (the latter using a human shaker), moving pedestrian load and response measurement and crowd tracking. There are many more possibilities.

Keywords: footbridge dynamic monitoring modal testing wireless sensor pedestrian loading

\section{Introduction}

With structural safety of footbridges well managed, the concern for designers has for the last two decades shifted to vibration serviceability. There have been some classic vibration serviceability failures [1-2] to perform adequately under human dynamic loads usually with large crowds, while a number of footbridges are lively even for a single pedestrian. While there are now advanced design codes for footbridge dynamic design [3-4] dealing with group and crowd loading as well as lateral vibrations, the reality is often different to design and experimental assessment of the as-built structure is frequently required. The Vibration Engineering Section (VES) at the University of Exeter has been involved in experimental studies of a significant number of footbridges either to confirm adequate performance or to provide information and guidance on such structures that are lively in one way or another.

These experimental studies have uncovered two cases of synchronous lateral excitation and a number of footbridges with performance on the edge of acceptability. In such cases even with code compliance, retrofit may be deemed necessary. In all these cases the experimental studies centred on system identification, or more specifically experimental modal analysis, to identify modal frequencies, damping ratios, shapes and masses, often with some form of proof testing by individuals or crowds. Where a footbridge has been retrofitted (with some form of damper) further experimental evaluation is required to prove effectiveness. 
A good example of the traditional modal test is the study of the Helix Bridge, [5], Fig. 1, commissioned by Land Transport Authority, Singapore and carried out as by VES spinout-out Full Scale Dynamics Ltd. This iconic structure functions as both footbridge and grandstand (for viewing events in Marina Bay) so required evaluation using a hybrid experimental-analytical approach against dual serviceability criteria.
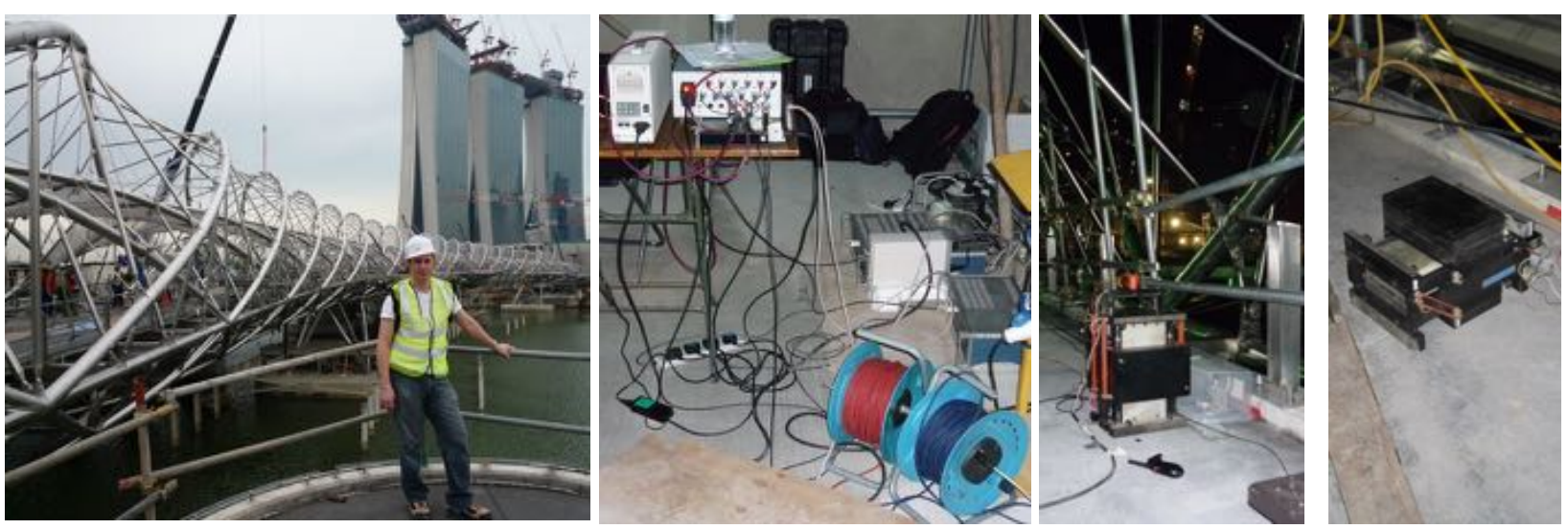

Figure 1 Modal test of Helix Bridge, Singapore using two shakers

The modal test was a major exercise involving costly air-freighting of $125 \mathrm{~kg}$ of sensors and cabling and local procurement of two APS electro-dynamic shakers totalling $150 \mathrm{~kg}$ of heavy shakers. The procedure worked flawlessly, providing high quality data for a modal model that showed the bridge to be serviceable under the most onerous loading. However considering the logistical complexity of the 13,500 mile round trip, it is natural to consider a procedure that provides slightly reduced level or quality of data but at much lower cost. Hence the objective of the research described here was to evaluate:

1) The capability of lightweight wireless inertial measurement units (IMUs) for synchronous acceleration measurements at multiple locations on a footbridge for monitoring or operational modal analysis;

2) The capability of the same sensors to provide indirect estimation of ground reaction forces (GRFs) due to walking or jumping to excite bridge response in specific footbridge vibration modes;

3) The feasibility of using IMU data for input (GRF) and output (footbridge response) to generate frequency response functions of adequate quality to recover all modal parameters including modal mass.

Up to this point only vertical loads and responses are considered although the technology is being extended to lateral loads. The footbridge chosen for studying the methodology using IMUs is a footbridge in Exeter whose behaviour is entirely satisfactory while exhibiting behaviour useful for a number of research projects.

\section{Measurement of walking and jumping GRFs}

At present, in 2017, formal guidance on design for vertical GRFs due to walking and jumping derive from laboratory measurements on force plates. The most widespread data for walking, found in all British floor design guidance [5-7] in fact derive from a single study where single footfalls obtained by test subjects passing over a single force plate were taken as perfectly periodic at pacing rate. These assumed-periodic time series were used to derive dynamic load factors (DLFs) that are the ratios of Fourier line amplitude at the pacing rate or fundamental, and it's multiples or harmonics, to pedestrian weight. These DLFs are typically provided up to the fourth harmonic, but are not so widely used for footbridges, where a range of DLFs for the fundamental component are provided in various design guides.

\subsection{Direct measurement of GRFs}

It has been established [8] using an instrumented treadmill to record GRFs for continuous walking that far from being perfectly periodic, walking GRFs are narrow band random processes. Following on from this result, an EPSRC-funded three-axis treadmill was used to record a comprehensive set of three-axis GRFs for a range of test subjects walking and jogging at a range of pacing rates [9-10] in order to establish better GRF models for design. Even these GRFs are imperfect representations of the forces generated during walking since they are constrained by operation of a treadmill and were generated in the laboratory rather than on the full-scale structure. 
The only way to obtain real-world walking GRFs is by direct measurement in the real world, actually on footbridges and this requires a different kind of instrumentation. Possibilities for direct measurement include multiple force plates and instrumented insoles. Jumping forces can be directly measured in situ using a force plate, but our experience is that a heavy force plate which needs to be placed on level ground and which requires its own power supply and signal conditioning is not well suited to fieldwork.

For walking GRFs, given the difficulty in using even a single force plate for jumping the challenges of full instrumenting a footbridge with force plates are prohibitive due to cost, data acquisition and logistics of temporary retrofit. Instrumented insoles, also expensive, have promise [11] despite reservations about measuring the total GRF in world coordinates.

\subsection{GRF measurement using optical motion capture}

To avoid such limitations indirect measurement of GRF has been studied using motion capture technology. Classical motion capture systems use either passive markers (Vicon, Qualisys) or active markers (Coda Motion). Measurements on test subjects instrumented with arrays of Coda active markers while walking on a treadmill or jumping on a force plate [11-12] have shown that application of Newton's Second Law summing inertia forces via markers attached to body segments is a reliable proxy for direct measurement. To obtain GRFs via inertia forces, body segment masses from cadavers of similar size to test subjects are scaled by double-differentiated displacement data and summed over all markers.

While proving to be surprisingly reliable this approach has numerous disadvantages. Firstly while the motion capture systems are not restricted to the small area of a force plate or treadmill, they can still measure only in limited volumes and do not work outside in daylight. Second, visibility of key markers can be obscured compromising the inertia force summation and thirdly, double-differentiating can lead to numerical errors. Figure 2 shows an attempt to use a Coda active marker system to track pedestrians wearing multiple active markers on the reinforced polymer footbridge at EMPA laboratory, Zurich [13]. The exercise was only partially successful mainly due to problems of infra red interference with capturing marker locations using cameras positioned either side of the bridge.
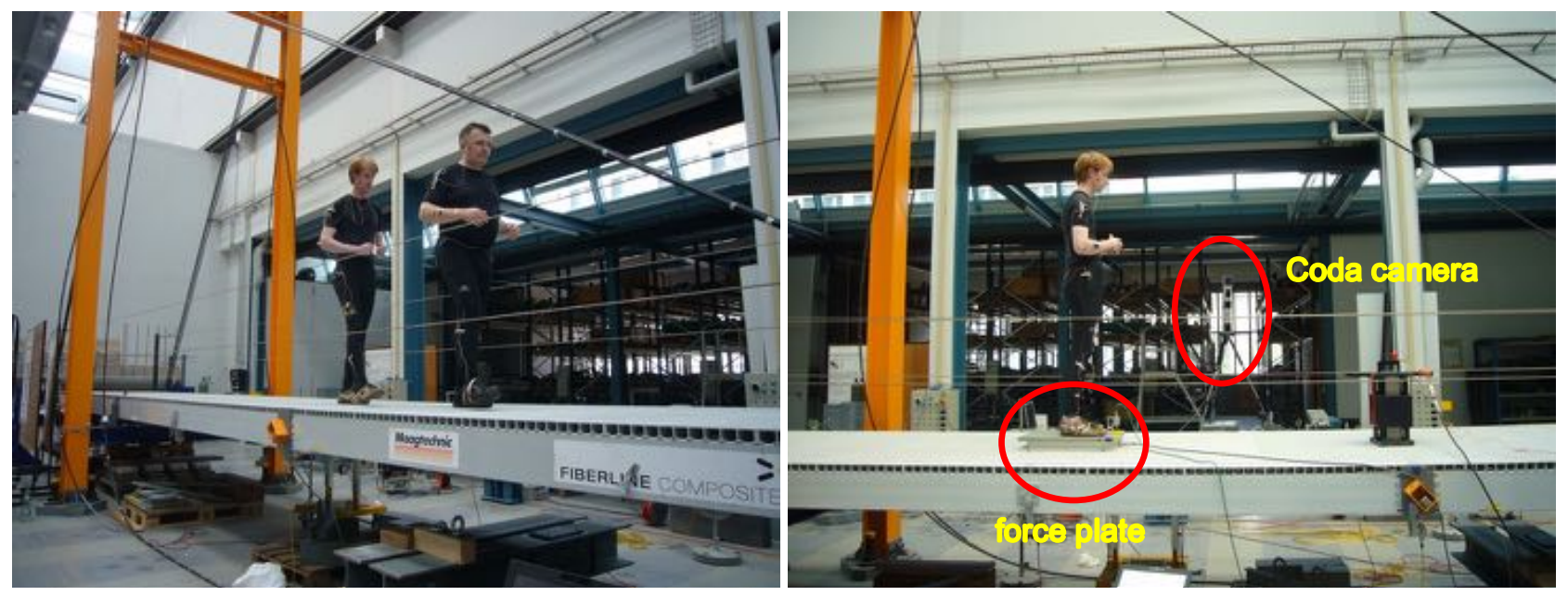

Figure 2 Coda system at EMPA laboratory measuring forces and response due to walking and jumping.

An alternative marker free tracking system was also explored, using technology first applied to gesture recognition in human-computer interfaces [14], but this also faced limits of field of view and robustness.

\subsection{GRF measurement using inertial measurement units}

Due to limitations of tracking with optical motion tracking restricting capability outside a laboratory environment, capabilities of wireless accelerometer arrays were evaluated. At that time the available products were from XSens, APDM and Shimmer. Rather than using Xsens system [15] we first investigated the more economic Shimmer system before acquiring a set of APDM Opal inertial measurement units (IMUs) [16]. These have since been used in an extensive series of measurements using them directly as wireless accelerometers as well as for indirect recovery of GRFs through measurements on human subjects. 
IMUs function in a radically different way to optical markers. Whereas optical markers acquire position in 3D space from which acceleration data may be obtained by double-differentiation, IMUs provide acceleration data directly. Unfortunately the raw acceleration data are acquired in the local coordinate system (LCS) of the IMU, and require conversion to a reference world coordinate system (WCS) for GRF recovery. In addition to accelerometers Opals contain a triaxial gyroscope and magnetometers so that using quaternions (a form of coordinate transformation) acceleration signals can be recovered referenced to a North, East, Up WCS. This conversion is totally unambiguous for recovering vertical WCS signals.

Initially it was expected that multiple Opals would be required to provide adequate representation of GRFs for a single person, leading to a series of evaluations, Figure 3, using force plate and treadmill.

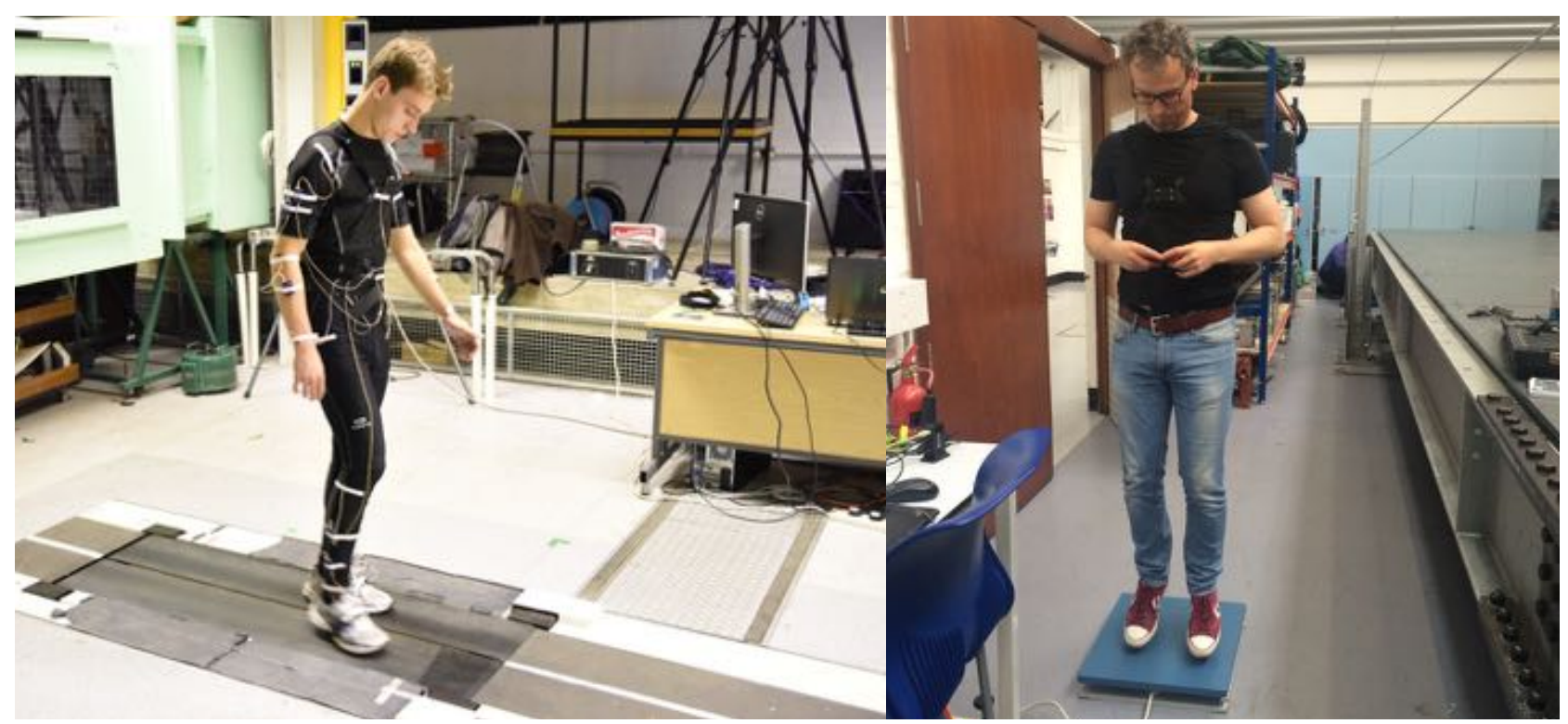

Figure 3 Experiments validating use of Opals for GRF recovery during walking and jumping.

Walking GRF measurements (Figure 3, left) which initially aimed to compare capability of Coda and Opal systems quickly showed that a single Opal IMU attached to the 7th cervical vertebra (C7) could recover the GRFs component at the fundamental pacing frequency with an accuracy of $15 \%$ at $90 \%$ confidence level [17]. Likewise, a single Opal at $\mathrm{C} 7$ could recover the fundamental component of jumping GRF with good accuracy. Figure 4 compares time histories acquired directly (treadmill or force plate) with vertical acceleration signals from an IMU attached to C7, and the FFTs for the direct force signal with those obtained using vertical acceleration signals from IMUs attached at C7, lower back, navel and sternum (breast bone). These are examples for a single person (subject) walking or jumping at a single frequency and which in this case show reasonable agreement with variation depending on which location on the body is used. It is clear that not only are jumping forces much greater but also simpler compared to walking forces, so that representation using IMU signals might work better for jumping forces.

Close inspection of IMU data usually shows components at higher frequencies that reflect in greater differences between FFTs at higher harmonic frequencies for direct and indirect measurements. The FFT plots are line amplitudes of GRFs and are used to determine dynamic load factors (DLFs) through division by subject weight; in the walking example the DLF would be approximately 0.2 while in the jumping example the DLF would be approximately 1.3. In the two examples the FFTs are not a single line because the walking and jumping time histories are in fact narrow band random processes rather than perfectly periodic as assumed in the design guides. To obtain an equivalent to the perfectly periodic model, rather than taking the single maximum value an alternative to calculating the DLF would be to take the square root of sum of square of spectral line amplitudes close to multiples of the activity rate $f_{p}$, say $f_{p} \pm 5 \%$. 

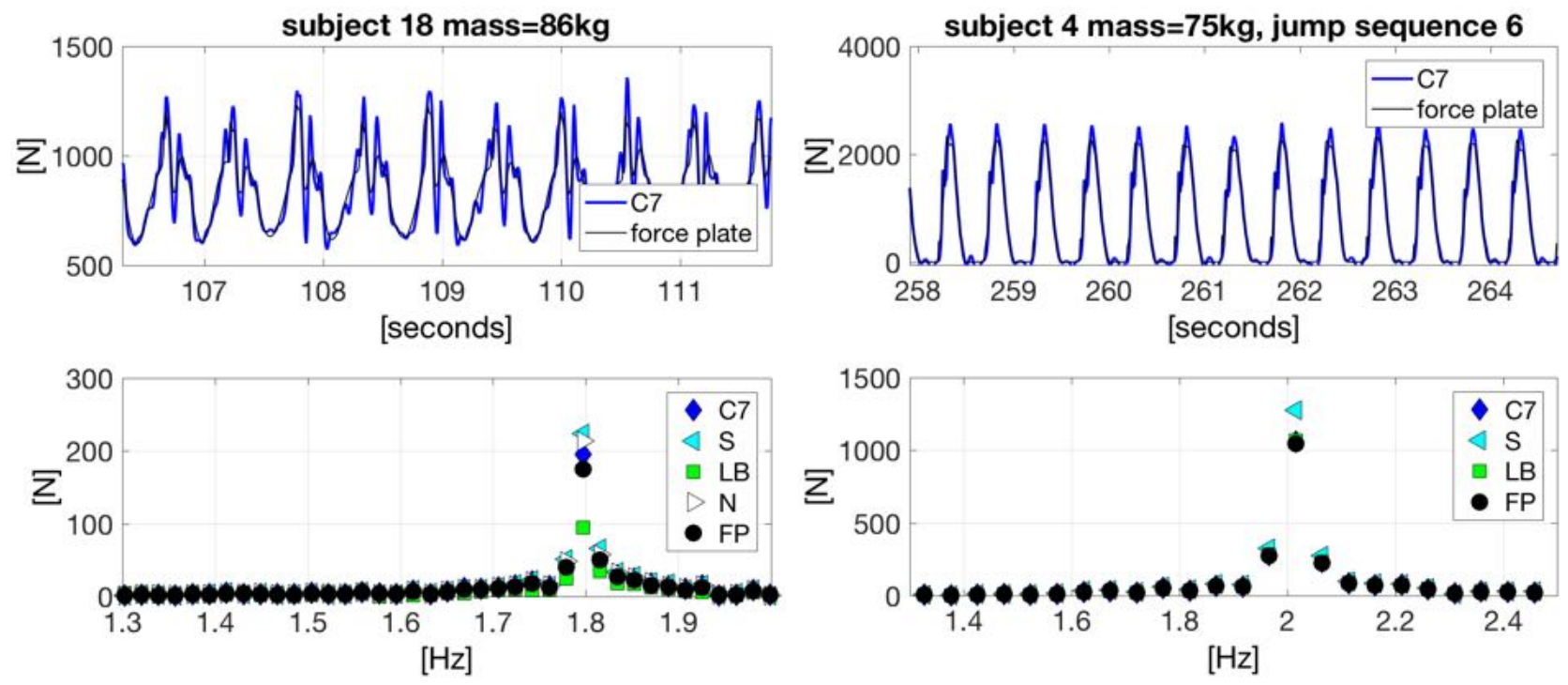

Figure 4 Sample time series and first harmonic FFT comparisons for direct and indirect GRF measurement using mass-scale acceleration. Left: using treadmill for walking. Right: using force plate for jumping.

To demonstrate the performance of IMUs for a range of subjects for a range of $f_{p}$ and different IMU locations, Figure 5 plots the ratio of DLFs computed for $f_{p} \pm 5 \%$. These comparisons are remarkable; they show that for the controlled conditions of artificially constrained walking or jumping attaching a single IMU to the subject's neck at $\mathrm{C} 7$ will allow for indirect estimation of GRFs for at least the fundamental frequency component.
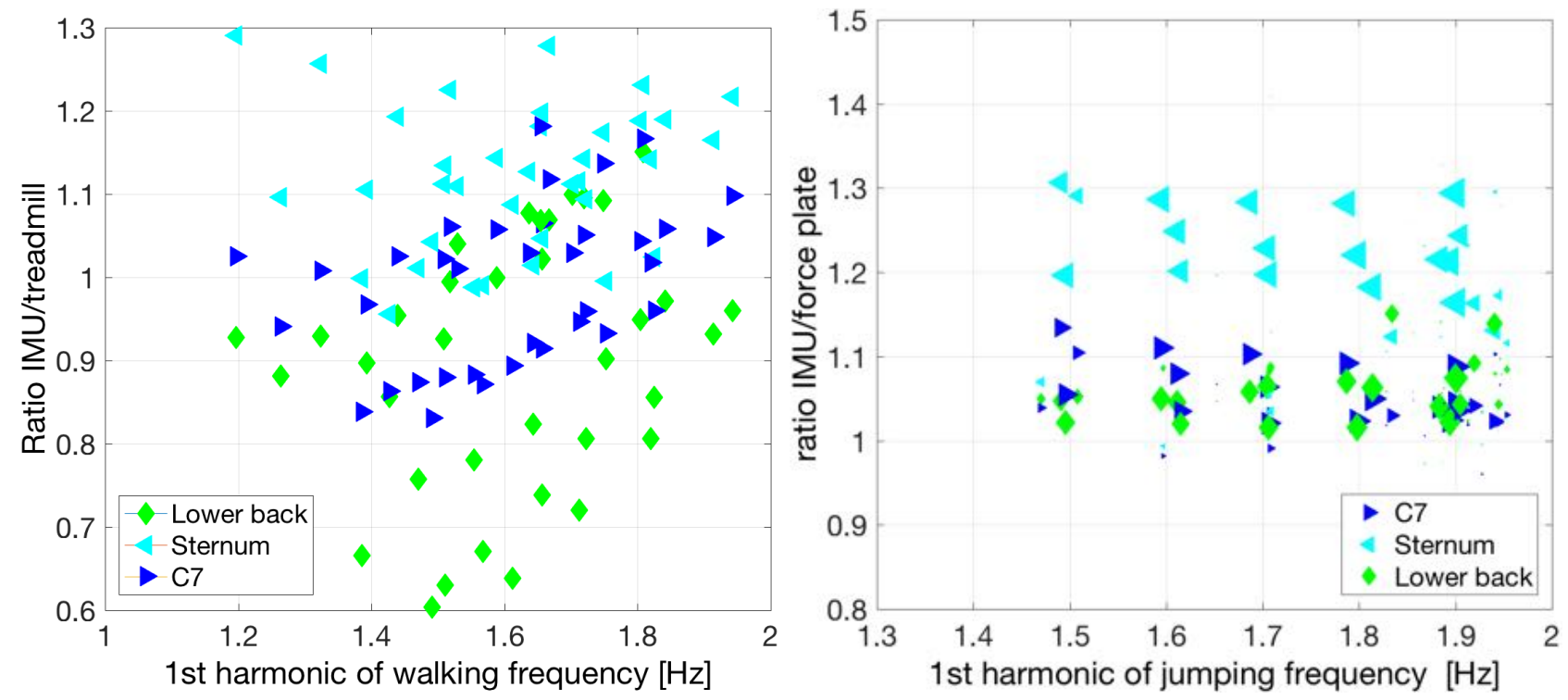

Figure 5 Comparison of dynamic load factors from IMU and direct measurements for fundamental frequency.

This rather surprising result has significant consequences for studying footbridge performance since it allows for in-situ estimation of (walking or jumping) GRFs for as many pedestrians and as many structure response points as there are IMUs available. In principle a pair of IMUs would suffice to study the response of a footbridge to a single pedestrian.

\section{Application of IMU to footbridge system identification}

\subsection{Exeter footbridges}

The research on IMU capability began during a study on pedestrian synchronisation within an undergraduate student project, using three candidate footbridges around Exeter, Figure 6. 


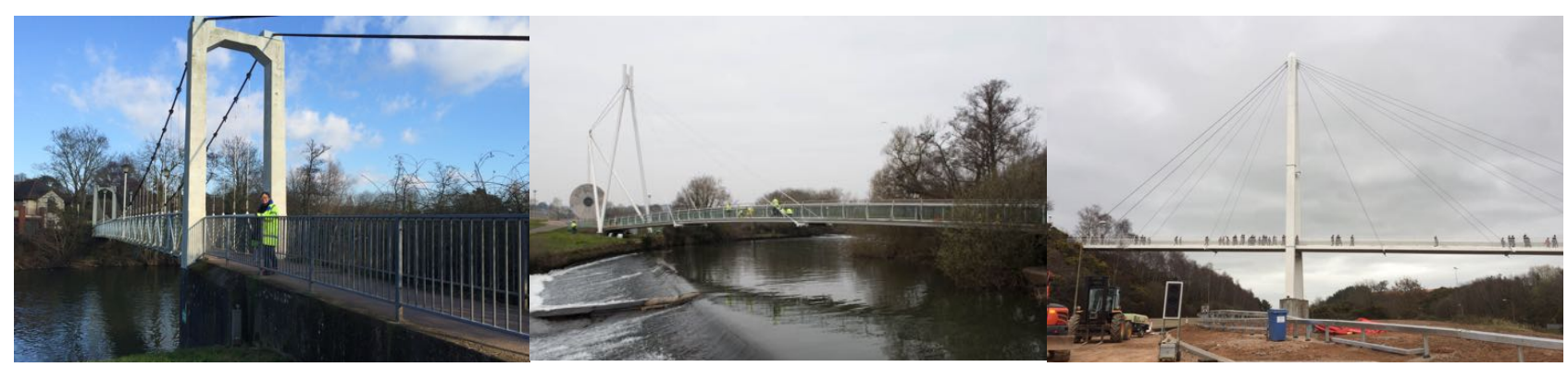

Figure 6 Exeter footbridges: Trews Weir, Millers Crossing and Baker Bridge

Trews Weir is a very lively suspension bridge, so much that supports partially lift off during strong vibrations resulting in strongly nonlinear behaviour with a complex pattern of mode shapes. Miller's Crossing cablestayed bridge appears to be an ideal candidate for strong pedestrian-induced response but a set of tuned mass dampers installed by Devon County Council limits significant pedestrian-induced response. Finally Baker Bridge had neither limitation. Despite being the largest and heaviest of the three structures and having the lowest fundamental natural frequency it proved to exhibit strong (but code compliant) pedestrian-induced vibrations. It was thus an excellent candidate for investigation of response to pedestrian dynamic loads.

\subsection{Baker Bridge}

Baker Bridge (Figure 7) provides cycle and pedestrian access between Digby and Sowton railway station and Sandy Park Stadium, the home ground of Exeter Chiefs, the English Rugby Union champions for 20162017 season. During match days the bridge experiences heavy pedestrian traffic and demonstrates a lively dynamic response, but even a single jogger or pedestrian can generate strong response.

The bridge has a single A-shaped $42 \mathrm{~m}$ tower supporting the continuous $109 \mathrm{~m}$ deck via seven pairs of cables. Four cable pairs support a long front span on the (South) stadium side, two cable pairs support a short back span on the North side and one pair connects to a counterbalance at the north abutment.
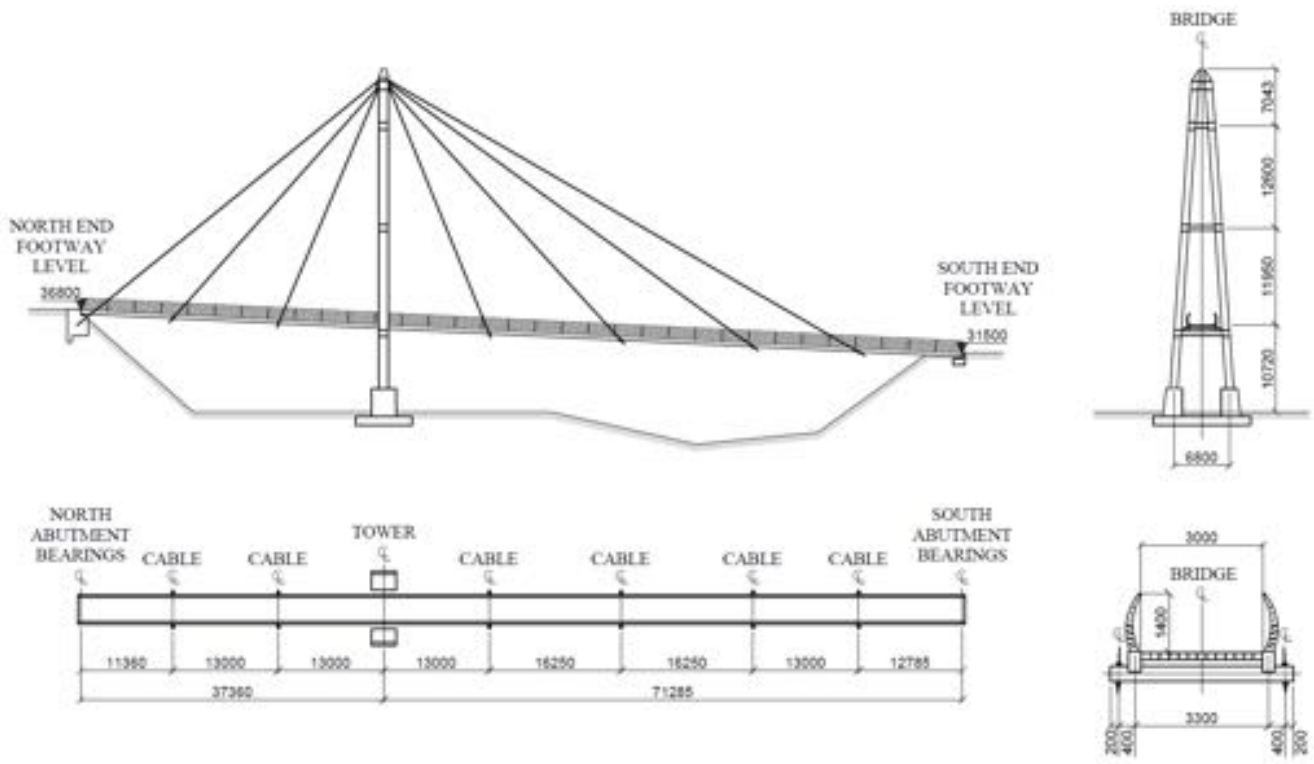

\section{Figure 7 Baker Bridge}

The tower uprights are steel rectangular hollow section (RHS) $1200 \times 600 \mathrm{~mm}$ supported on sitting on

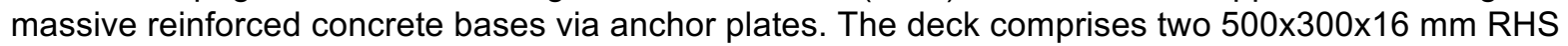
longitudinal beams with transverse $150 \times 150 \times 5 \mathrm{~mm}$ square hollow section (SHS) beams at $3.1 \mathrm{~m}$ centres.

Rolled steel angle sections $100 \times 100 \times 8 \mathrm{~mm}$ welded to the RHS beams provide support for the $120 \mathrm{~mm}$ in-situ reinforced concrete walkway, which is also secured to the SHS beams by $\phi 19 \times 74 \mathrm{~mm}$ shear studs at 150 $\mathrm{mm}$ centres. The six cable pairs are secured to $324 \times 16 \mathrm{~mm}$ circular hollow section that support the longitudinal beams. The total mass of the bridge is estimated as 150 tonnes. 
Figure 8 shows synchronization experiments using Opal IMUs. The IMUs were attached to the shoes of six student test subjects of which one was designated as leader with other students not knowing the aim of the test. The aim of the study was to investigate relationship of footfall timing with position in the group (of six) relative to the leader. Also being investigated was any influence of bridge vertical vibration on footfall timing. In order to plan the tests for best effect it was necessary to first identify approximate modal properties i.e. mode shape, frequency and damping ratio, and an attempt was made to use IMUs for the identification. The results from the synchronization study are at the time of writing being written up for publication.
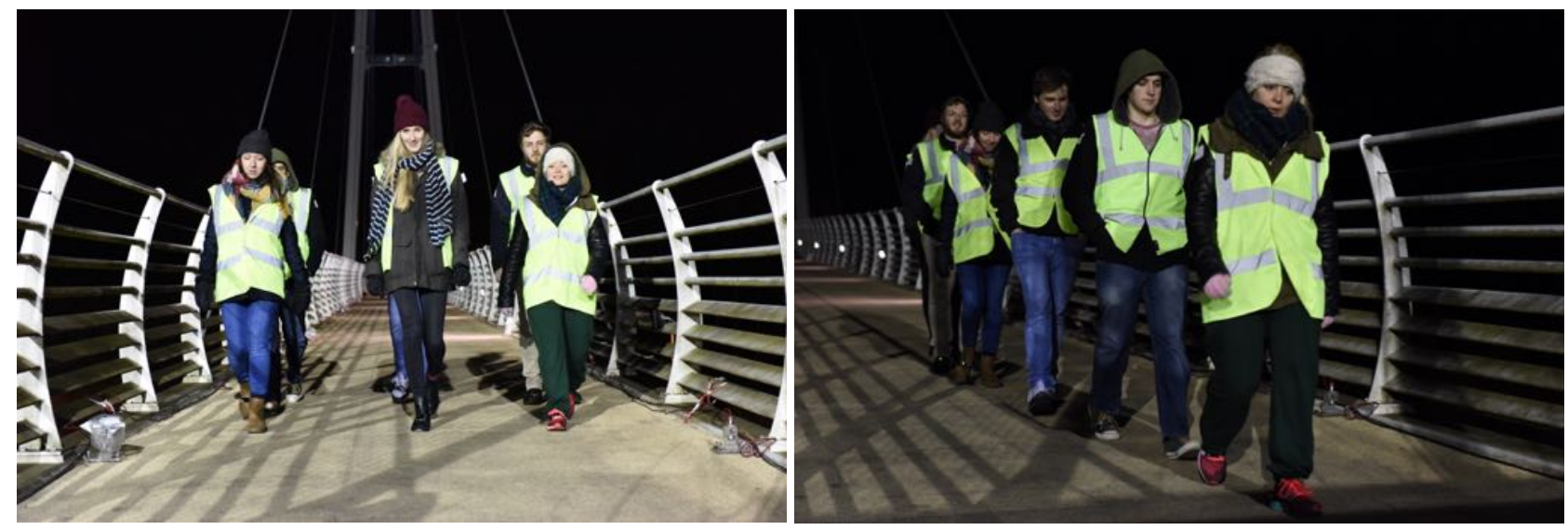

Figure 8 Pedestrian synchronisation studies with Baker Bridge

\subsection{Ambient vibration test}

MEMS accelerometers typically do not achieve the sub $\mu \mathrm{g} / \mathrm{VHz}$ noise floors usually required for ambient vibration testing of civil infrastructure. Since the noise floor for the Opal IMU is quoted as $128 \mu \mathrm{g} / \mathrm{VHz}$ it was not certain that the resolution would be adequate for clear identification. In the event, the strong response of the bridge to excitation by wind and the occasional pedestrian (Figure 9 upper) proved adequate to identify the vibration modes with frequencies in the range of pedestrian pacing rates using a sequence of measurements in which a pair of IMUs were kept at reference locations while others roved to other measurement points along the bridge. The NExT-ERA procedure was used to identify a set of six modes, two of which are shown in Figure 9 lower. Damping ratios due to NExT-ERA procedures are expected to be higher than values obtained by free or forced vibration measurements.
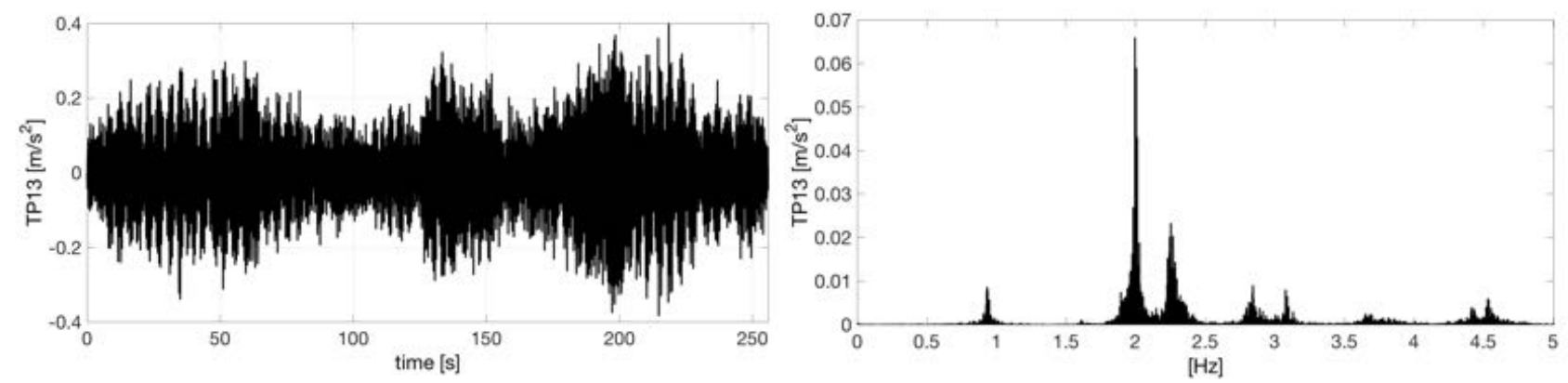

Mode 1: $f_{\mathrm{n}}=0.939 \mathrm{~Hz}, \zeta_{\mathrm{n}}=0.34 \%$

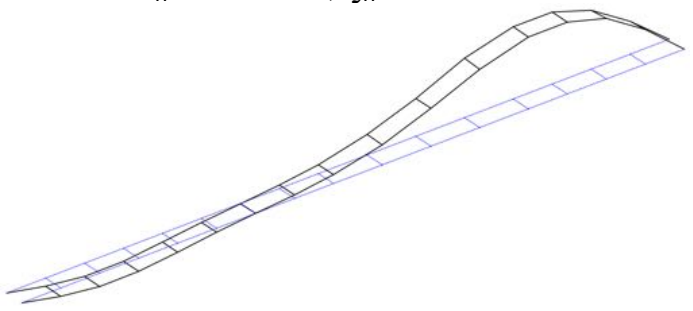

Mode 3: $f_{\mathrm{n}}=2 \mathrm{~Hz}, \zeta_{\mathrm{n}}=0.35 \%$

Figure 9 Ambient vibration test results using Opals: Sample time series and FFTs, and two mode shapes. 


\subsection{Instrumented jumping tests to identify modal masses}

Ambient vibration testing is traditionally used with very large, tall or long structures such as office towers and suspension bridges where shaker excitation is not feasible. Unlike forced vibration testing with known excitation, modal mass cannot be measured directly and both frequency and damping estimates suffer from significant bias variance errors due to the random nature of the excitation and the limitations of the identification procedure. Hence IMUs were used to measure simultaneously the excitation force provided by a short sequence of jumping and the footbridge response. Excitation was measured using an IMU attached either to sternum, lower back or $\mathrm{C} 7$ of the test subject and bridge response using an IMU on the deck. Jumping force was applied and response measured where the modal ordinate reaches a maximum response according to the mode shapes in Figure 9. Identification of Mode 1 was via a $74 \mathrm{~kg}$ test subject (student) using an IMU on the sternum jumping and recording deck response at the maximum modal ordinate. The result is illustrated in Figure 10. The raw acceleration data (top left) are for jumping at a range of frequencies corresponding to the identified modes, showing a sequence of partial decays. The first of these is then bandpassed from $0.7 \mathrm{~Hz}$ to $1.2 \mathrm{~Hz}$ and sternum acceleration scaled by body mass in tonnes (lower left). Both signals are exponentially windowed before fitting a circle to the resulting frequency response function (FRF) as the ratio of response to force (right). The damping is heavily over-estimated, by a known value, almost $1 \%$, due to the exponential windowing, but the modal mass is a reasonable estimate of the true value.

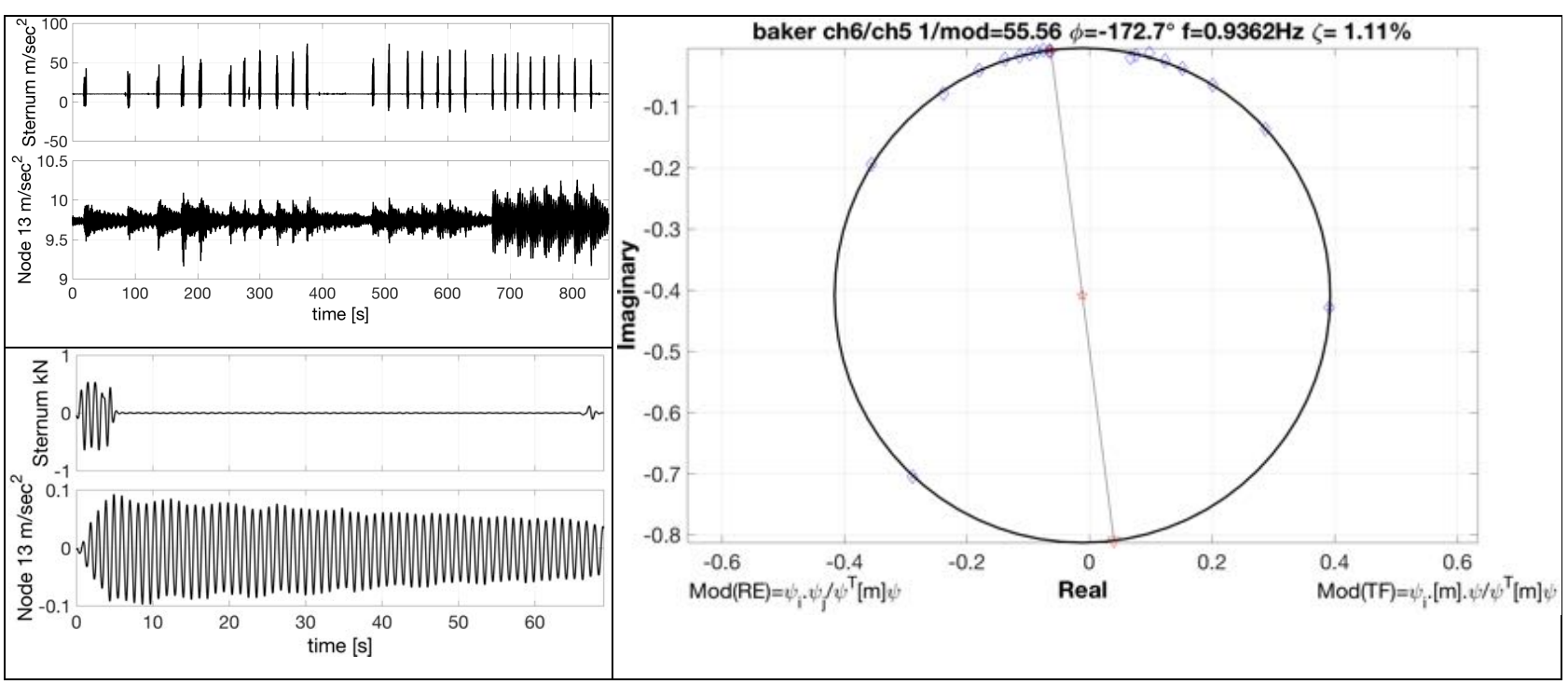

Figure 10 Identification of mode 1. Raw data for jumping and response for a range of frequencies starting at $0.9 \mathrm{~Hz}$ (upper left); Band-pass filtered accelerations, sternum values scaled by body mass (lower left); circle fit to frequency response function between exponentially windowed time histories (right). The circle plots imaginary vs. real components of the FRF.

The measurement illustrated in Figure 10 preceded the calibration exercise of Figure 5 that identified $\mathrm{C} 7$ as the optimal IMU location. A more controlled exercise including an IMU at $\mathrm{C} 7$ and also taking the trouble to deploy a force plate in-situ is illustrated in Figure 11. The exercise concentrated on identifying modes susceptible to pedestrian excitation, and did not include Mode 1. The strong windowing for Figure 10 data was needed due to leaving too short a period $T=60 \mathrm{~s}$ for measuring the jumping sequence and free decay. The resulting frequency spacing $d f=1 / T$ does not provide good resolution of the frequency response function (FRF) that changes rapidly with frequency due to the very low damping and adding known damping via the exponential window is a classical method to improve resolution. To avoid this requirement the second exercise shown in Figure 11 used $\mathrm{T}=160 \mathrm{~s}$.

In Figure 11 the time histories for vertical forces obtained by scaling IMU vertical acceleration by body mass are presented without filtering. The force plate signal is the reference and also includes the self-inertia. The higher frequency components for sternum and lower back do not have significant effect on the quality of the FRF around Mode 3 frequency $(2 \mathrm{~Hz})$, but $\mathrm{C} 7$ appears to best match the force plate signal. The curve fit to the Nyquist circle of imaginary vs. real components of the FRF has to be adjusted to account for the modal ordinate of the response measurement point, and the resulting modal mass estimate is 60.6 tonnes. 

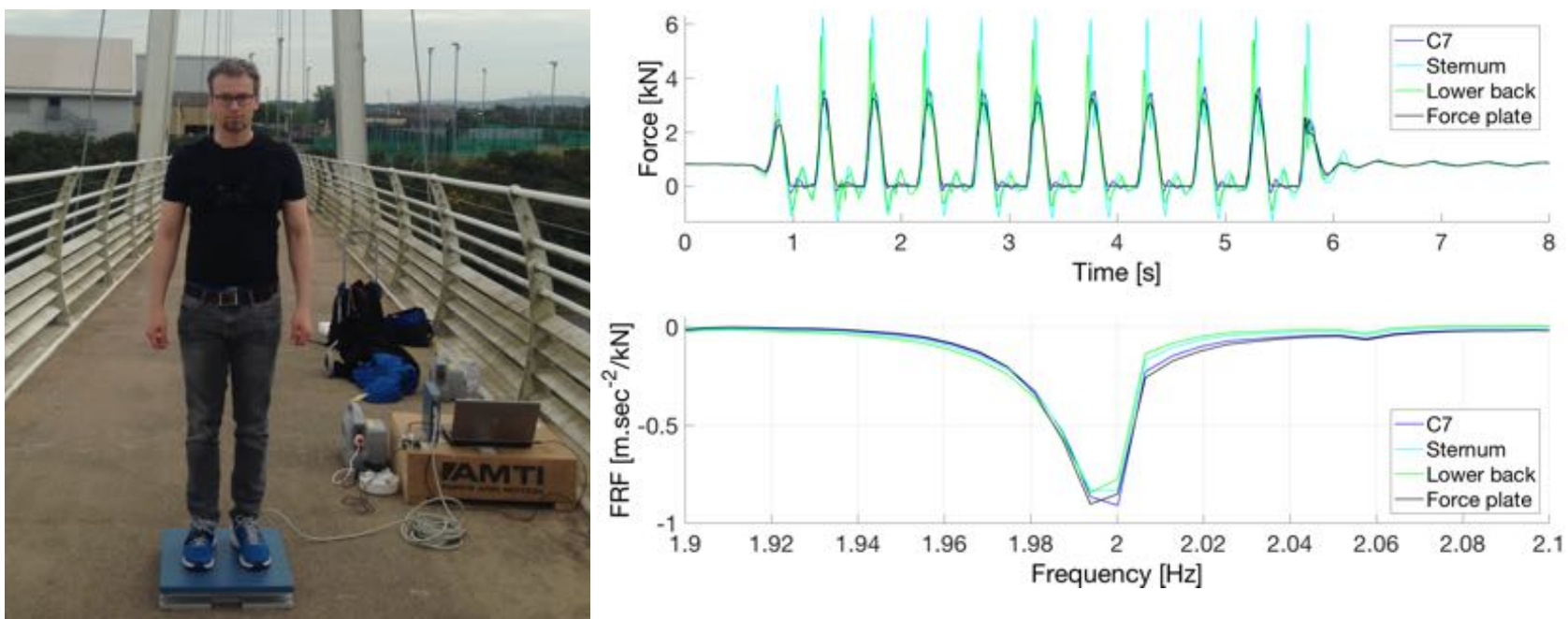

Figure 11 Identification of Mode 3 using IMUs at C7, sternum and lower back and directly using a force plate. Frequency response function (FRF) lower right is the imaginary part obtained by averaging over four jumps with 160-second duration $T$.

\section{Discussion and conclusions}

Beginning with the need to provide reliable measurements of continuous ground reaction forces (GRFs) for structure design and moving to the need to measure GRFs in-situ, we have determined that a single strategically located inertial measurement unit (IMU) can provide surprisingly accurate representation of the forces relevant for pedestrian excitation of a footbridge. The result has subsequently been used for modal testing of a footbridge in Singapore (with low logistical costs) and represents a powerful capability for fast modal identification of lightweight structures having natural frequencies around the fundamental harmonic frequency of walking. In fact the Singapore test showed that identification is possible at the second harmonic.

We continue to study the capability for force identification under a range of circumstances, including the medio-lateral forces generated deliberately or otherwise to engage horizontal vibrations of footbridges.

\section{Acknowledgements}

We are grateful to Devon County Council for granting permission to carry out measurements on the Exeter footbridges. We also thank the legion of students who assisted throughout the research and various academic colleagues including Chris Middleton, Vito Racic and Aleksandar Pavic who have also contributed to the research leading to the findings reported here.

\section{References}

[1] Dallard P. R., Fitzpatrick T., Flint A., Low A., Smith R., "The Millennium Bridge, London: problems and solutions," Struct. Eng., Vol. 79, no. 8, pp. 15-17, 2001.

[2] Dziuba P., Grillaud G., Flamand O., Sanquier S., and Tetard Y., "La passerelle Solferino:

Comportement dynamique (Solferino bridge: Dynamic behaviour)," Bull. ouvrages métalliques, no. 1, pp. 3457, 2001.

[3] Setra, "Guide méthodologique passerelles piétonnes (Technical guide Footbridges: of footbridges under pedestrian loading)," Setra, 2006.

[4] British Standards Institution, UK National Annex to Eurocode 1: Actions on structures - Part 2: Traffic loads on bridges, no. 1. 2003.

[5] Pavic A. and Willford M. R., "Vibration Serviceability of Post-tensioned Concrete Floors - CSTR43 App G," Appendix G in Post-Tensioned Concrete Floors Design Handbook - Technical Report 43. Concrete Society, Slough, UK, pp. 99-107, 2005. 
[6] Willford M. R. and Young P., A Design Guide for Footfall Induced Vibration of Structures - CCIP-016. Slough: The Concrete Centre, 2006.

[7] Smith A. L., Hicks S. J., and Devine P. J., Design of floors for vibration - A new approach SCI P354, Revised Ed, SCI P354. The Steel Construction Institute, 2009.

[8] Brownjohn J. M. W., Pavic A., and Omenzetter P., "A spectral density approach for modelling continuous vertical forces on pedestrian structures due to walking," Can. J. Civ. Eng., Vol. 31, no. 1, pp. 6577, Jan. 2004.

[9] Racic V. and Brownjohn J. M. W., "Mathematical modelling of random narrow band lateral excitation of footbridges due to pedestrians walking," Comput. Struct., Vol. 90-91, pp. 116-130, Jan. 2012.

[10] V. Racic, J. M. W. Brownjohn, and A. Pavic, "Dynamic loading factors of individual jogging forces," in 4th ECCOMAS Thematic Conference on Computational Methods in Structural Dynamics and Earthquake Engineering, June 2013.

[11] Racic V., Pavic A., and Brownjohn J. M. W., "Modern facilities for experimental measurement of dynamic loads induced by humans: A literature review," Shock Vib., Vol. 20, no. 1, pp. 53-67, 2013.

[12] Racic V., Brownjohn J. M. W., and Pavic A., "Reproduction and application of human bouncing and jumping forces from visual marker data," J. Sound Vib., Vol. 329, no. 16, pp. 3397-3416, 2010.

[13] Zivanovic S., Feltrin G., Mottram J. T., and Brownjohn J. M. W., "Vibration Performance of Bridges Made of Fibre Reinforced Polymer," in IMAC32, pp. 1-8, 2014.

[14] Zheng F., Shao L., Racic V., and Brownjohn J. M. W., "Measuring human-induced vibrations of civil engineering structures via vision-based motion tracking," Measurement, Vol. 83, pp. 44-56, Apr. 2016.

[15] Van Nimmen K., Lombaert G., Jonkers I., De Roeck G., and Van den Broeck P., "Characterisation of walking loads by 3D inertial motion tracking," J. Sound Vib., Vol. 333, no. 20, pp. 5212-5226, Sep. 2014.

[16] Brodie M. A. D., Beijer T. R., Canning C. G., and Lord S. R., "Head and pelvis stride-to-stride oscillations in gait: validation and interpretation of measurements from wearable accelerometers," Physiol. Meas., Vol. 36, no. 5, pp. 857-872, May 2015.

[17] Bocian M., Brownjohn J. M. W., Racic V., Hester D., Quattrone A., and Monnickendam R., "A framework for experimental determination of localised vertical pedestrian forces on full-scale structures using wireless attitude and heading reference systems," J. Sound Vib., Vol. 376, pp. 217-243, 2016. 